\title{
EFECTO DE PYRACLOSTROBIN EN LA PRODUCCIÓN DE PAPA CULTIVAR DIACOL CAPIRO
}

Fecha de recepción: 06 de marzo de 2014 • Fecha de aceptación: 17 de mayo de 2014

\section{EFFECT OF PYRACLOSTROBIN IN CROP PRODUCTION OF DIACOL CAPIRO POTATO VARIETY}

Jennifer Andrea Patiño Cardona', José Miguel Cotes Torres², Jorge Andrés Ramírez González

\section{RESUMEN}

La gota de la papa causada por Phytophthora infestans (Mont.) de Bary, es una enfermedad que causa grandes pérdidas al cultivo, y requiere una frecuente aplicación de productos químicos. El pyraclostrobin, tiene un modo de acción que se basa en la inhibición de la respiración mitocondrial para proteger de enfermedades fúngicas a diferentes cultivos y además a mostrado ser un producto que mejora la acumulación de asimilados en la planta. Este trabajo tuvo como objetivo evaluar el efecto bioestimulante del pyraclostrobin, para lo cual se aplicó el producto al tubérculo semilla y al follaje, evaluándose siete diferentes momentos de aplicación; se utilizó un testigo sin utilización de pyraclostrobin. Se encontró que, en general, son preferibles las aplicaciones solamente al follaje que al follaje y al tubérculo semilla. El pyraclostrobin aplicado al follaje en aporque y floración obtiene incrementos de materia seca superiores al control sin aplicación de 117\%, 25\% y 75\% para hojas, tallos y tubérculos, respectivamente.

Palabras clave: Phytophthora infestans, Gota de la papa, Bioestimulante.

1. Agr. MSc. en Ciencias Agrarias. Universidad Nacional de Colombia - Sede Medellín - Facultad de Ciencias Agrarias - Departamento de Ciencias Agronómicas - Cll. 59A No 63-20 - Núcleo El Volador, Medellín - Colombia. e-mail: jeapatinoca@unal.edu.co

2. Agr. MSc. DSc. Profesor Asociado. Universidad Nacional Colombia - Sede Medellín - Facultad de Ciencias Agrarias - Departamento de Ciencias Agronómicas - Cll. 59A No. 63-20 - Núcleo El Volador, Medellín - Colombia. e-mail: jmcotes@unal.edu.co

3. Agr. MSc. Representante R\&D UIC Occidente BASF Química Colombiana S.A. Calle 99 No. 69C-32, Bogotá - Colombia. e-mail: jorge.ramirez@partners.basf.com. 


\begin{abstract}
The potato late blight caused by Phytophthora infestans (Mont.) de Bary, is an important disease because produce a heavy losses to the crop, and it requires frequent application of chemical products. The pyraclostrobin, has a mode of action based on the mitochondrial respiration inhibition to protect crops from different fungal diseases, also has been shown to be a product that enhances the accumulation of assimilates in the plant. This research was carried out to evaluate the biostimulant effect of pyraclostrobin in potato. The product was spray to seed tubers and foliage, being evaluated seven different spray moments, additionally a check treatment without pyraclostrobin was used. We found that, in general, sprays only to foliage are better than sprays to foliage and seed tuber. The pyraclostrobin spray to foliage in flowering and earthing up obtained increases in dry matter of $117 \%, 25 \%$ and $75 \%$ for leaves, stems and tubers, respectively.
\end{abstract}

Keywords: Phytophthora infestans, potato, late blight, plant regulation.

\title{
INTRODUCCIÓN
}

A nivel mundial, los mayores productores de papa son Asia y Europa, los cuales suministraron el 80\% de la producción en 2007. El primero consume casi la mitad del suministro de papa, con un consumo por persona de $24 \mathrm{~kg}$ año-1 comparado al de los europeos (88 kg persona-1 año-1 $^{-1}$ ), el consumo mas bajo está en América Latina (21 kg persona-1 $\mathrm{año}^{-1}$ ) y África (14 kg persona ${ }^{-1}$ año-1). La cuna de la papa está en América del Sur, pero esta región tiene el nivel más bajo de producción, de menos de 16 millones de toneladas en 2007 (FAO, 2009).

El cultivo de la papa es el más importante de las zonas altas de Colombia, donde se cultivan alrededor de 128.701 ha las cuales tienen un rendimiento promedio de aproximadamente 2'272.772 ton año-1 (Rivera et al., 2010). La especie Solanum tuberosum spp. andigena ocupa el primer lugar dentro de las explotaciones de clima frío. Antioquia ocupa el cuarto lugar en producción de papa en el país, con un área de 14.015 ha y un rendimiento promedio de 17,3 t ha-1 $^{-1}$ Agrocadenas, 2006).
La producción mundial de papa ha aumentando a una tasa media anual del 4,5\% en los últimos 10 años, y ha superado el crecimiento de la producción de muchos otros importantes productos alimentarios en los países en desarrollo (FAO, 2009).

Al cultivo de la papa, se encuentran vinculadas 90.000 familias campesinas en promedio. Es uno de los cultivos con mayor demanda de jornales al año y agroquímicos en el país (Herrera et al., 2000) para el control de una de las causas principales de pérdidas en producción, la gota o tizón tardío, causado por el oomycete Phytophthora infestans (Mont.) de Bary (Agrocadenas, 2006).

El tizón tardío se encuentra en casi todas las regiones del mundo, siendo más virulenta en zonas frías y húmedas. De igual forma afecta otros cultivos de la familia Solanaceae como el tomate (Vega, 2004). $P$. infestans es un patógeno que se puede propagar sexual y asexualmente, el primero proporciona un estado de supervivencia en condiciones adversas, y se producen unas estructuras llamadas oosporas. De 


\section{El mecanismo de acción del pyraclostrobin está enfocado en la} inhibición de la cadena transportadora de electrones, actuando en el complejo III sobre el sitio de reducción de la ubiquinona oxidasa (Qol), entre el citocromo by c1, inhibiendo asíla respiración (Parrado et al., 2010; FRAC, 2013;), lo que ocasiona una reducción significativa de la producción de ATP (Bartlett et al., 2002).

forma asexual las estructuras de reproducción son los esporangios, en una lesión de la enfermedad se puede producir hasta 300.000 esporangios, que al madurar se dispersan gracias a las corrientes de viento e infectan nuevas plantas hospedantes (Henfling, 1987; Jaramillo, 2003). La enfermedad es muy destructiva en aquellas áreas con épocas de alta humedad relativa y temperaturas bajas, condiciones que permiten la proliferación del patógeno, lo que incrementa los costos de producción, siendo esenciales las aplicaciones de fungicidas para proteger el cultivo (Agrios, 2005).

El pyraclostrobin (2-[1-(4-clorofenil)pirazol-3-iloximetil]$\mathrm{N}$-metoxicarbanilato de metilo; PY), de la familia de las estrobilurinas, sólo o en combinación con otros fungicidas presenta un modo de acción que se basa en la inhibición de la respiración mitocondrial para proteger de enfermedades fúngicas a diferentes cultivos como: uva, fresa, pimiento, tomate, papa y diferentes cereales y caducifolios (Balba, 2007; Schnabel y Crisosto, 2008; Esteve-Turrillas et al., 2011; Xiangwei et al., 2012).

El mecanismo de acción del pyraclostrobin está enfocado en la inhibición de la cadena transportadora de electrones, actuando en el complejo III sobre el sitio de reducción de la ubiquinona oxidasa (Col), entre el citocromo b y $c_{1}$, inhibiendo así la respiración (Parrado et al., 2010; FRAC, 2013;), lo que ocasiona una reducción significativa de la producción de ATP (Bartlett et al., 2002).
En trabajos llevados en campo para el control de $P$. infestans encontraron que parcelas tratadas con una mezcla de pyraclostrobin + metiram presentaron elevados niveles de control en el cultivo de la papa (Töfoli et al., 2003) y cuando se trataba con una mezcla de pyraclostrobin + metiram + una bacteria benéfica (Bacillus subtilis) + diferentes fungicidas protectantes y sistémicos (mancozeb y dimetomorf), la enfermedad también fue controlada eficazmente (Navia et al., 2012). Con plantas de cebada tratadas con pyraclostrobin solamente, no se desarrollaron síntomas de manchas fisiológicas en hojas (efecto fisiológico), lo que indica que algunas reacciones de bloqueo se están desarrollando internamente en la planta de radicales que son opuestos al estrés oxidativo, presentando una contribución a la tolerancia al estrés, lo cual puede evidenciarse cuando la actividad de la peroxidasa es evaluada (Köhle et al., 2002a), el cual se presenta por las condiciones desfavorables del ambiente o por la acción de una enfermedad cualquiera ocasionando la formación de radicales, especialmente de formas reactivas de oxigeno, lo cual incrementa el potencial oxidativo (Bartosz, 1997; Wingsle et al., 1999). De igual forma Karadimos et al. (2005), confirman la efectividad de pyraclostrobin contra Cercospora beticola en plantas de remolacha azucarera incluso a tasas de aplicación muy bajas y realizadas luego de que los síntomas de la enfermedad aparecieron. 
Cualquier planta que se encuentre en contacto con un fungicida en este caso con el pyraclostrobin, absorbe una cierta cantidad de este, lo que produce un cambio en el metabolismo de la planta, y genera un mayor crecimiento e incrementos significativos en la producción. Esto se debe a que se presentan efectos adicionales en la fisiología del cultivo que generan a una influencia positiva en la producción (Köhle et al., 2002b). Otros efectos han sido encontrados por Binotto et al. (2010), donde plantas de soya con aplicaciones de pyraclostrobin han aumentado su tasa fotosintética en un 3 y 10\% en relación con los testigos, tan sólo tres horas después de la aplicación.

El objetivo del presente trabajo fue evaluar el efecto bioestimulante del pyraclostrobin en el cultivo de papa cultivar DIACOL Capiro en condiciones agroecológicas del corregimiento de Santa Elena, Antioquia.

\section{MATERIALES Y MÉTODOS}

\section{Condiciones experimentales}

Las parcelas experimentales se realizaron entre marzo y septiembre de 2013, en el municipio de Medellín, corregimiento de Santa Elena, en el Centro Agropecuario Paysandú de La Universidad Nacional de Colombia a una altura de $2.575 \mathrm{msnm}$, las coordenadas geográficas son $6^{\circ} 12^{\prime} \mathrm{LN}$ y $75^{\circ} 30^{\prime} \mathrm{LO}$, la temperatura media diaria durante el ciclo del cultivo fue de $14,6^{\circ} \mathrm{C}$ y una precipitación acumulada de $691,6 \mathrm{~mm}$.

En el experimento se utilizó semilla certificada del cultivar DIACOL Capiro, producida en noviembre de 2012. Las prácticas de manejo del cultivo fueron las tradicionalmente realizadas por el agricultor haciendo especial énfasis en la nutrición y sanidad del cultivo.

\section{Diseño experimental}

En un diseño de bloques completos al azar con cuatro repeticiones se establecieron 15 tratamientos en un arreglo factorial 7X2 (7 momentos de aplicación al follaje $\times 2$ formas de aplicación) y un tratamiento adicional como testigo utilizando para el control de gota productos protectantes y sistémicos diferentes del pyraclostrobin (Tabla 1), no se adicionó un testigo sin aplicación ya que la enfermedad en Antioquia es muy agresiva y si no es controlada la planta muere rápidamente después de emergencia. La unidad experimental fue conformada por cuatro surcos de $10 \mathrm{~m}$ de largo con 25 plantas por surco, la distancia entre plantas fue de 0,4 m y entre surcos de 1,0 $\mathrm{m}$ para un área total de $40 \mathrm{~m}^{2}$ por unidad experimental, los dos surcos de los extremos fueron barreras para evitar la deriva, en los dos surcos centrales se realizaron las evaluaciones, dejando a cada extremo 1 m como barrera. La dosis aplicada en todos los tratamientos con pyraclostrobin fue de 0,6 kg ha-1.

Los tratamientos tuvieron un control rutinario de gota de la papa ( $P$. infestans), el cual consistió de aplicaciones con fungicidas protectantes (ametoctradina, mancozeb, metiram y chlorathalonil) intercalados con fungicidas sistémicos (dimetomorf, mefonoxam, propamocarb, cimoxanilo y ametoctradin) (Figura 1) dependiendo de la edad del cultivo e infección, luego de la emergencia de las plantas.

\section{Variables evaluadas}

Se evaluó la severidad de la gota de la papa en hojas, realizando mediciones semanales, con el uso de la escala propuesta por el CIP (Henfling, 1987) (Tabla 2). Estas lecturas fueron realizadas durante 11 semanas consecutivas desde los 25 días después de la siembra hasta los 95 días después de la siembra. Se determinó el área bajo la curva de progreso de la enfermedad (ABCPE) mediante la siguiente expresión (Campbell y Madden, 1990):

$$
A B C P E=\sum_{i=1}^{n-1}\left(\frac{y_{i}+y_{i+1}}{2}\right)\left(t_{i+1}-t_{i}\right)
$$


Tabla 1. Tratamientos aplicados para evaluar el efecto de la aplicación del pyraclostrobin en el tubérculo semilla y en siete momentos de aplicación al follaje, en el cultivo de papa (Solanum tuberosum).

\begin{tabular}{|c|c|c|c|c|c|c|c|}
\hline Tratamiento & & & Descripción & & & & \\
\hline 1 & Aplicación a la semilla & + & Deshierbe & & & & \\
\hline 2 & Aplicación a la semilla & + & Aporque & & & & \\
\hline 3 & Aplicación a la semilla & + & Floración & & & & \\
\hline 4 & Aplicación a la semilla & + & Deshierbe & + & Aporque & & \\
\hline 5 & Aplicación a la semilla & + & Deshierbe & + & Floración & & \\
\hline 6 & Aplicación a la semilla & + & Aporque & + & Floración & & \\
\hline 7 & Aplicación a la semilla & + & Deshierbe & + & Aporque & + & Floración \\
\hline 8 & Sin aplicación a la semilla & + & Deshierbe & & & & \\
\hline 9 & Sin aplicación a la semilla & + & Aporque & & & & \\
\hline 10 & Sin aplicación a la semilla & + & Floración & & & & \\
\hline 11 & Sin aplicación a la semilla & + & Deshierbe & + & Aporque & & \\
\hline 12 & Sin aplicación a la semilla & + & Deshierbe & + & Floración & & \\
\hline 13 & Sin aplicación a la semilla & + & Aporque & + & Floración & & \\
\hline 14 & Sin aplicación a la semilla & + & Deshierbe & + & Aporque & + & Floración \\
\hline 15 & Testigo sin pyraclostrobin & & & & & & \\
\hline
\end{tabular}

Donde " $t$ " es el tiempo de cada lectura, " $y$ " el porcentaje de follaje afectado en cada lectura y " $n$ " el número de mediciones de la enfermedad.

Por otro lado, se realizaron evaluaciones para la determinación del efecto bioestimulante del pyraclostrobin, por lo cual se realizó un muestreo a los 106 días después de la siembra, cuando las plantas se encontraban en floración y engrosamiento de tubérculos. La muestra se tomó de dos plantas completas por unidad experimental y fueron Ilevadas al Laboratorio de Fisiología Vegetal de la Universidad Nacional de Colombia - Sede Medellín, donde se dividió la planta en sus órganos componentes, hojas, tallos, estolones, tubérculos, flores y frutos, y se ubicaron de forma separada en la estufa de secado a $80^{\circ} \mathrm{C}$ hasta alcanzar peso seco constante. El peso seco y fresco de cada muestra se determinó con ayuda de una balanza de precisión.

La cosecha se realizó a los 200 días después de la siembra. Se realizaron mediciones de peso fresco de tubérculos y se clasificaron en cuatro categorías: Muy grande: $>90$ mm, Grande: $65-90$ mm, Mediano: 45-64 mm y Pequeña: 30-44 mm., utilizando unas zarandas de madera con esos diámetros. 
Tratamientos con aplicación al tubérculo semilla

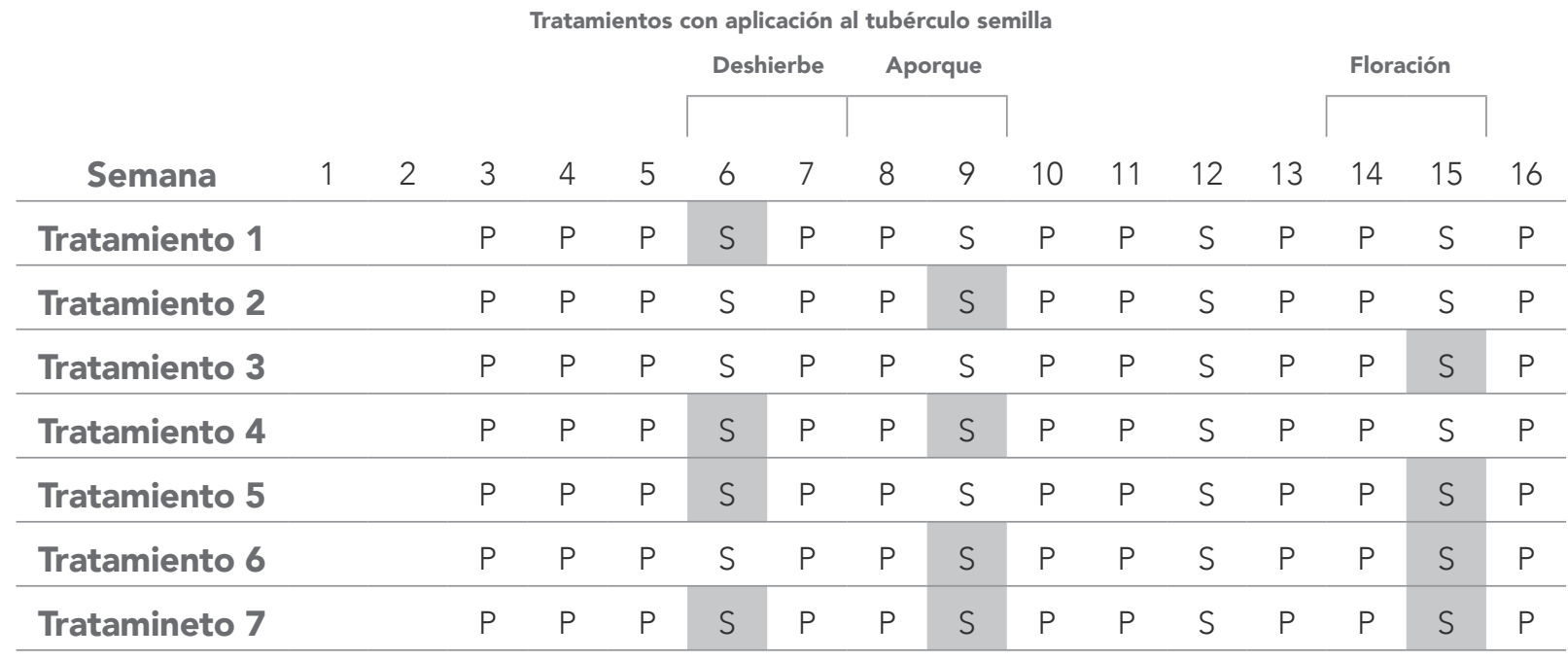

Tratamientos sin aplicación al tubérculo semilla

\begin{tabular}{|c|c|c|c|c|c|c|c|c|c|c|c|c|c|c|c|c|}
\hline \multirow[b]{2}{*}{ Semana } & \multirow[b]{2}{*}{1} & \multirow[b]{2}{*}{2} & \multirow[b]{2}{*}{3} & \multirow[b]{2}{*}{4} & \multirow[b]{2}{*}{5} & \multicolumn{2}{|c|}{ Deshierbe } & \multicolumn{2}{|c|}{ Aporque } & \multirow[b]{2}{*}{10} & \multirow[b]{2}{*}{11} & \multirow[b]{2}{*}{12} & \multirow[b]{2}{*}{13} & \multicolumn{2}{|c|}{ Floración } & \multirow[b]{2}{*}{16} \\
\hline & & & & & & 6 & 7 & 8 & 9 & & & & & 14 & 15 & \\
\hline Tratamiento 8 & & & $P$ & $P$ & $P$ & $\mathrm{~S}$ & $P$ & $P$ & $\mathrm{~S}$ & $P$ & $P$ & $\mathrm{~S}$ & $\mathrm{P}$ & $P$ & $\mathrm{~S}$ & $\mathrm{P}$ \\
\hline Tratamiento 9 & & & $P$ & $P$ & $P$ & $\mathrm{~S}$ & $P$ & $P$ & $S$ & $P$ & $P$ & $\mathrm{~S}$ & $P$ & $P$ & $\mathrm{~S}$ & $P$ \\
\hline Tratamiento 10 & & & $P$ & $P$ & $P$ & $\mathrm{~S}$ & $P$ & $P$ & $\mathrm{~S}$ & $P$ & $P$ & $\mathrm{~S}$ & $P$ & $P$ & $\mathrm{~S}$ & $P$ \\
\hline Tratamiento 11 & & & $P$ & $P$ & $P$ & $\mathrm{~S}$ & $P$ & $P$ & $\mathrm{~S}$ & $P$ & $P$ & $S$ & $P$ & $P$ & $\mathrm{~S}$ & $\mathrm{P}$ \\
\hline Tratamiento 12 & & & $P$ & $P$ & $P$ & $\mathrm{~S}$ & $P$ & $P$ & $\mathrm{~S}$ & $P$ & $P$ & $\mathrm{~S}$ & $P$ & $P$ & $\mathrm{~S}$ & $\mathrm{P}$ \\
\hline Tratamiento 13 & & & $P$ & $P$ & $P$ & $\mathrm{~S}$ & $P$ & $P$ & $\mathrm{~S}$ & $P$ & $P$ & S & $P$ & $P$ & S & $P$ \\
\hline Tratamiento 14 & & & $P$ & $P$ & $P$ & $S$ & $P$ & $P$ & $\mathrm{~S}$ & $P$ & $P$ & S & $\mathrm{P}$ & $P$ & S & $\mathrm{P}$ \\
\hline Tratamiento 15 & & & $P$ & $P$ & $P$ & $S$ & $P$ & $P$ & $S$ & $P$ & $P$ & $S$ & $P$ & $P$ & $S$ & $P$ \\
\hline
\end{tabular}

\begin{tabular}{|cl|}
\hline $\begin{array}{c}\text { Tratamientos: } \\
\text { (1-14): }\end{array}$ & $\begin{array}{l}\mathrm{P}=\text { Aplicación con producto Protectante } \\
\mathrm{S}=\text { Aplicación con producto Sistémico diferente a pyraclostrobin }\end{array}$ \\
$\begin{array}{cl}\text { Tratamiento } \\
\text { 15: }\end{array}$ & Sin aplicación con Pyraclostrobin \\
\hline
\end{tabular}

Figura 1. Aplicaciones de fungicidas intercalados entre protectantes y sistémicos de acuerdo al estado fenológico de la planta de papa (Solanum tuberosum). Destacados con gris están las aplicaciones de pyraclostrobin. 
Tabla 2. Clave para la evaluación de P. infestans en papa bajo condiciones de campo (Tomado de Henfling, 1987).

\begin{tabular}{|c|c|c|c|}
\hline \multirow{2}{*}{$\begin{array}{l}\text { Valores } \\
\text { escala del } \\
\text { CIP }\end{array}$} & \multicolumn{2}{|c|}{ P. infestans (\%) } & \multirow{2}{*}{ Síntomas } \\
\hline & Media & Límites & \\
\hline 1 & 0 & & No se observa $P$. infestans \\
\hline 2 & 2,5 & Trazas $<5$ & P. infestans presente. Máximo 10 lesiones por planta \\
\hline 3 & 10 & $5<15$ & $\begin{array}{l}\text { Las plantas parecen sanas, pero las lesiones se pueden ob- } \\
\text { servar fácilmente al observar de cerca. No se observa una } \\
\text { área afectada o destruida mayor a } 20 \text { foliolos. }\end{array}$ \\
\hline 4 & 25 & $15<35$ & $\begin{array}{l}\text { P. infestans se observa con facilidad en las plantas. Alre- } \\
\text { dedor del } 25 \% \text { del área foliar esta cubierto por lesiones o } \\
\text { destruido. }\end{array}$ \\
\hline 5 & 50 & $35<65$ & $\begin{array}{c}\text { Las plantas lucen verdes, pero todas están afectadas por el } \\
\text { patógeno, las hojas inferiores muertas. Alrededor del 50\% } \\
\text { del área foliar está destruida. }\end{array}$ \\
\hline 6 & 75 & $65<85$ & $\begin{array}{l}\text { Las plantas se observan verdes con manchas pardas. Alre- } \\
\text { dedor del } 75 \% \text { de la planta afectada. Las hojas de la mitad } \\
\text { inferior de la planta está destruida. }\end{array}$ \\
\hline 7 & 90 & $85<95$ & $\begin{array}{l}\text { Las plantas no están predominantemente verdes ni pardas. } \\
\text { Solo las hojas superiores están verdes. Muchos tallos tienen } \\
\text { lesiones externas. }\end{array}$ \\
\hline 8 & 97,5 & $95<100$ & $\begin{array}{c}\text { La planta se ve parda. Unas cuantas hojas superiores aún } \\
\text { presentan coloración verde. La mayoría de los tallos están } \\
\text { lesionados o muertos. }\end{array}$ \\
\hline 9 & 100 & & Todas las hojas y los tallos están muertos. \\
\hline
\end{tabular}

\section{Análisis estadístico}

Para la variable ABCPE se realizó un análisis de varianza con los 15 tratamientos. Para las variables de materia seca a los 106 días y producción a los 200 días por categorías se calculó, por bloque, la relación o cociente entre la respuesta obtenida por el tratamiento con pyraclostrobin (del 1 al 14) y el tratamiento sin pyraclostrobin, es decir el tratamiento 15.

$$
R_{i}=\frac{y_{i}}{y_{15}} \quad i=1, \ldots, 14(2)
$$

A esas relaciones se les obtuvo el análisis de varianza respectivo con los 14 tratamientos con pyraclostrobin. Para conocer las diferencias con el control sin aplicación de pyraclostrobin se realizó una prueba t-student para la hipótesis de que la relación sea igual a $1\left(H_{0}: R_{i}=1\right)$. 
Tabla 3. Relación de la materia seca de hojas, tallos, raíces y tubérculos relativa al testigo sin aplicación de pyraclostrobin a los 106 días después de la siembra. Valores superiores a 1 son mejores que el testigo. Los valores destacados en negro y cursiva son estadísticamente significativos $\left(H_{0}\right.$ : $R_{i}=1$ $\mathrm{p}<0,05)$. Las aplicaciones de pyraclostrobin en el follaje se realizaron al momento de la deshierba (D), aporque (A), floración (F) y sus combinaciones.

\begin{tabular}{|c|c|c|c|c|c|}
\hline & Tratamiento & Hojas & Tallos & Raíz & Tubérculo \\
\hline 1 & Semilla+D & 2,1408 & 0,995 & 1,0752 & 1,0881 \\
\hline 2 & Semilla + A & 1,9818 & 0,806 & 1,1891 & 0,9166 \\
\hline 3 & Semilla+F & 1,5088 & 0,796 & 1,0825 & 1,0076 \\
\hline 4 & Semilla+D+A & 1,0894 & 0,874 & 1,4454 & 1,3351 \\
\hline 5 & Semilla+D+F & 1,4015 & 0,8341 & 0,7687 & 1,134 \\
\hline 6 & Semilla $+A+F$ & 1,5855 & 0,6494 & 0,9729 & 0,9039 \\
\hline 7 & Semilla+D+A+F & 1,734 & 1,052 & 1,2389 & 1,3272 \\
\hline 8 & $\mathrm{D}$ & 1,7674 & 0,7905 & 1,2888 & 1,4513 \\
\hline 9 & $A$ & 2,1589 & 1,2561 & 1,005 & 1,0508 \\
\hline 10 & $\mathrm{~F}$ & 1,7579 & 0,7952 & 0,541 & 1,3673 \\
\hline 11 & $D+A$ & 1,3017 & 1,0601 & 0,821 & 0,9732 \\
\hline 12 & $D+F$ & 2,4392 & 1,1459 & 1,0306 & 1,6009 \\
\hline 13 & $A+F$ & 2,1768 & 1,2551 & 0,971 & 1,75 \\
\hline 14 & $D+A+F$ & 1,6156 & 0,6383 & 1,2731 & 1,074 \\
\hline
\end{tabular}

\section{RESULTADOS Y DISCUSIÓN}

Los datos de ABCPE no mostraron diferencias significativas entre los tratamientos evaluados, lo que muestra que las rotaciones con mayor o menor uso del pyraclostrobin controlan la enfermedad tan eficientemente como con la utilización de otros productos para el control de la enfermedad como lo ha reportado en varios estudios (Robledo-Esqueda et al., 2012; Wang et al., 2014).

La acumulación de materia seca en hojas relativa al tratamiento sin utilización de pyraclostrobin muestra que, tres de los siete tratamientos que implican aplicación a la semilla presentaron diferencias significativas, mientras que cinco de los siete que utilizaron solamente aplicaciones al follaje fueron significativamente superiores al testigo sin pyraclostrobin (Tabla 3). Esto sugiere que la aplicación exclusiva al follaje, en general, presenta mejores resultados que la mezcla de aplicación a la semilla y luego al follaje. Es de notar que los tratamientos significativos acumularon casi el doble de materia seca en hojas que el testigo sin pyraclostrobin, mostrando esto que el pyraclostrobin tiene un efecto fisiológico en el cultivo ya que favorece la acumulación de carbohidratos en hojas.

Por otra parte, en general, la aplicación frecuente de más de dos veces consecutivas en el cultivo, 
disminuye el efecto bioestimulante del pyraclostrobin, como se puede observar en todos los casos que no fueron estadísticamente significativos del control. Sin embargo, la excepción a la regla la presenta el tratamiento donde se aplicó a la semilla, y en el deshierbe, aporque y floración $(\mathrm{D}+\mathrm{A}+\mathrm{F})$, que presentó una relación promedio de 1,734. La mayor acumulación de materia seca en hojas frente al control, sin aplicación de pyraclostrobin, se obtuvo cuando se aplicó solamente en el follaje en el aporque y en la floración, lo cual está asociado con un período de máxima elongación de los estolones e inicio de tuberización, durante el ciclo vegetativo del cultivo, donde la planta está acumulando carbohidratos para luego ser trasladados a los órganos almacenadores (Salisbury y Ross, 1992; Taiz y Zeiger, 2006), en este caso los tubérculos.

En los tallos, se encontró que en 10 de los 14 tratamientos evaluados no se presentaron diferencias estadísticamente significativas del control sin aplicación de pyraclostrobin, indicando que en general este producto no afecta la acumulación de materia seca en tallos (Tabla 3). Sin embargo hay cuatro excepciones. Cuando se aplica en semilla, al aporque y en la floración se presentó un efecto negativo, es decir, el tratamiento acumula menos materia seca de tallos que el control sin aplicación de pyraclostrobin. Igualmente ocurre cuando el producto fue aplicado al follaje en el aporque (A) y en aporque y floración $(A+F)$ donde se obtuvieron las mayores acumulaciones de materia seca en tallos (aproximadamente 25\%) frente al control. Estos tratamientos fueron de los que más incrementaron la acumulación de materia seca en hojas lo que permite concluir que tanto en las hojas como en los tallos se pueden estar acumulando carbohidratos para su posterior redistribución (Gregory, 1956). Por último la aplicación consecutiva en las tres etapas fenológicas presentó un efecto negativo en la acumulación de materia siendo esta del 63,83\% de la acumulación que se obtiene al no aplicar el pyraclostrobin en el cultivo.

En raíces 12 de los 14 tratamientos evaluados no mostraron diferencias significativas respecto al testigo sin aplicación de pyraclostrobin (Tabla 3). Aplicaciones del producto al follaje en floración (F) redujeron significativamente el peso seco de la raíz, mientras que la aplicación a la semilla, en el deshierbe y el aporque $(D+A)$, aumentó la acumulación de materia seca en raíces en un $44 \%$.

En tubérculos dos de los siete tratamientos donde se aplicó pyraclostrobin en la semilla presentaron diferencias estadísticamente significativas del control, mientras que en las aplicaciones al follaje cuatro de los siete tratamientos presentaron dicha significancia (Tabla 3). Esto indica que, en general, las aplicaciones a la semilla son menos efectivas que las aplicaciones

\section{En tubérculos dos de los siete tratamientos donde se aplicó pyraclostrobin en la semilla presentaron diferencias estadísticamente significativas del control, mientras que en las aplicaciones al follaje cuatro de los siete tratamientos presentaron dicha significancia.}




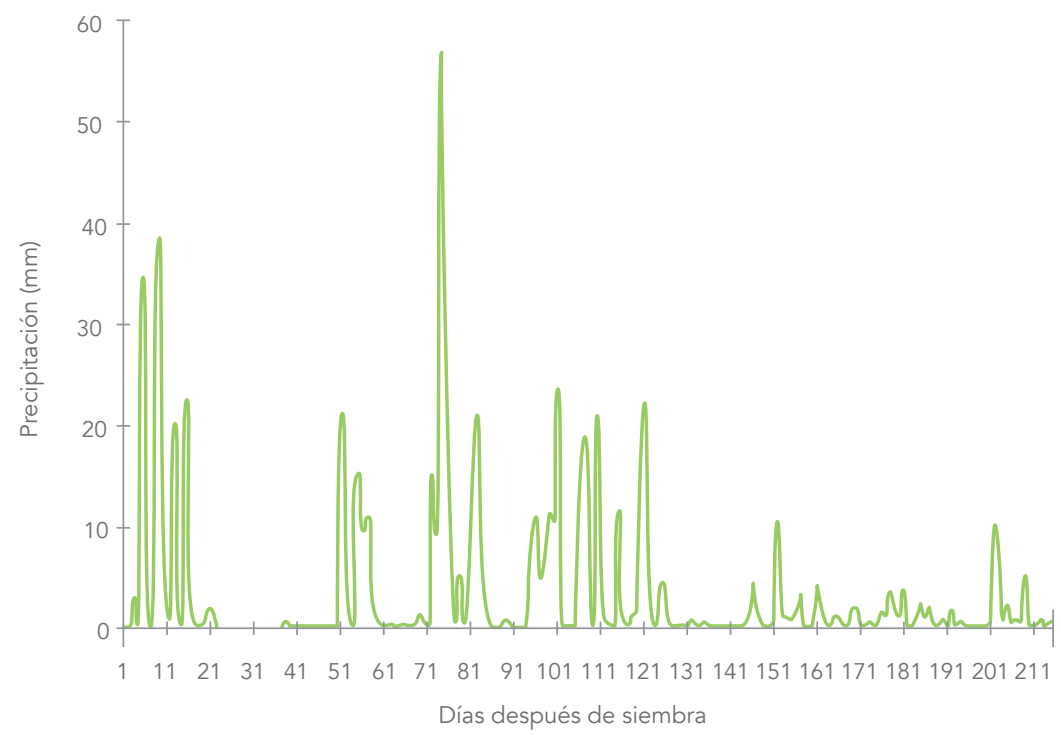

Figura 2. Precipitación diaria en milímetros $(\mathrm{mm})$, durante todo el ciclo del cultivo. Datos proporcionados por la estación climática cerca al ensayo.

exclusivamente al follaje, para incrementar la acumulación de materia seca de tubérculos, lo que concuerda con los resultados que se obtuvieron para la acumulación de materia seca en hojas. Es de notar que el tratamiento de pyraclostrobin a la semilla seguido de aplicaciones al follaje en deshierbe y aporque ( $D+A)$, incrementaron significativamente la materia seca de raíces y tubérculos, frente al tratamiento control, lo cual indica que estas plantas podrían estar mejor preparadas para soportar condiciones adversas del suelo como estrés hídrico o patógenos que afecten la raíz. Por otro lado el tratamiento de aplicación del producto a la semilla seguido de aplicaciones al follaje en deshierbe, aporque y floración, mostraron incrementos de materia seca en hojas y tubérculos, significativamente superiores que el control sin pyraclostrobin, indicando que este tratamiento fortalece la parte aérea de la planta y aumenta la producción potencial del cultivo. Este efecto, es decir, el aumento simultaneo de materia seca en hojas y tubérculos, es igualmente observado en los cuatro tratamientos que fueron significativos en aplicaciones solamente al foIlaje, es decir, aplicación al momento de la deshierba $(D)$, en floración $(F)$, en deshierba y floración $(D+F), y$ en apoque y floración ( $A+F)$. Nótese que el muestreo se realizó a los 90 días después de emergencia, que es la época en la que ya se tienen un $25 \%$ del peso final del tubérculo e inicia la redistribución acelerada de asimilados tales como carbohidratos, proteínas, minerales, entre otros (Moreno, 1985; Valbuena et al. 2010). Es importante destacar que la formación del tubérculo de papa es un proceso complejo, el cual está asociado con la finalización del desarrollo vegetativo y el comienzo del reproductivo, donde el estolón termina su crecimiento y comienza su llenado, el cual conlleva cambios genéticos, medioambientales, hormonales y de fotoperiodo (Ewing y Wareing, 1978; Gregory, 1956; Jackson, 1999; Stutte et al., 1996). Por esto, un fortalecimiento durante el ciclo del cultivo tanto en hojas y tallos como lo encontrado en los resultados con aplicación de pyraclostrobin es deseable para la buena producción. 
Este ensayo presentó rendimientos promedio por tratamiento entre 46,98 y $59,40 \mathrm{t} \mathrm{ha}^{-1}$, sin embargo, no se encontraron diferencias significativas con el control sin aplicación de pyraclostrobin. Según Dwelle (1985) altos rendimientos producidos en el cultivo de papa se atribuyen a la duración del área foliar y a la persistencia del dosel a medida que se acerca la senescencia de la planta, por lo que la aplicación del pyraclostrobin pudo haber significado altos rendimientos en este experimento. Sin embrago, en este ensayo se presentaron bajas precipitaciones (menores a $10 \mathrm{~mm} \mathrm{dí}^{-1}$ ) desde los 120 hasta los 200 días después de siembra (Figura 2), momento en el cual se encontraban en pleno llenado (Valbuena et al., 2010), por lo que el transporte de carbohidratos pudo verse afectado (Mares et al.,1985; Stutte et al., 1996; Viola et al., 2001) y así no presentar diferencias significativas del rendimiento final entre los tratamientos con pyraclostrobin y el control.

En conclusión, el uso del pyraclostrobin en un esquema de rotación de productos para el control de gota de la papa ( $P$. infestans), es tan eficiente como el uso de productos con otros ingredientes activos. En cuanto al efecto bioestimulate se puede afirmar que a los 106 días después de siembra en general es preferible las aplicaciones solamente al follaje que al follaje y al tubérculo semilla. El pyraclostrobin aplicado al follaje en aporque y floración obtiene incrementos de materia seca superiores al control sin aplicación de $117 \%, 25 \%$ y $75 \%$ para hojas, tallos y tubérculos, respectivamente.

\section{REFERENCIAS}

1. Agrios GN. 2005. Plant Pathology. Fifth edition. Academic Press, $9823 \mathrm{p}$.

2. Agrocadenas. 2006. La cadena de la papa: Una mirada global de su estructura y dinámica, 1991-2005 (Documento de trabajo N¹00). 38 p. Bogotá, Colombia.

3. Balba H. 2007. Review of strobilurin fungicide chemicals. Journal of Environmental Science and Health. Part. B, Pesticides, Food Contaminants, and Agricultural Wastes, 42(4): 441-451.

4. Bartlett DW, Clough JM, Godwin JR, Hall A Hamer M y Parr-Dobrzanski B. 2002. The strobilurin fungicides. Pest Management Science, 58(7): 649-662.

5. Bartosz G. 1997. Oxidative stress in plants. Acta Physiologiae Plantarum, 19(1): 47-64.

6. Binotto $E$, Dourado D, Vivian R, Brancher R, Pizzinato M, Massignam LF y Vilaça K. 2010. Efeito da aplicação de piraclostrobina na taxa fotossintética, respiração, atividade da enzima nitrato redutase e produtividade de grãos de soja. Bragantia, 69(4): 771-777.

7. Campbell CL y Madden LV. 1990. Introduction to plant disease epidemiology. WileyInterscience, NY. 532 pp.

8. Dwelle RB. 1985. Photosynthesis and photoassimilate partitioning. p. 35-58. En: Li PH (Ed.). Potato Physiology. Academic Press. Orlando, Florida: Elsevier. 
9. Esteve-Turrillas FA, Mercader JV, Agulló C, Abad-Somovilla A y Abad-Fuentes A. 2011. Development of immunoaffinity columns for pyraclostrobin extraction from fruit juices and analysis by liquid chromatography with UV detection. Journal of Chromatography. 1218(30): 4902-909.

10. Ewing EE y Wareing PF. 1978. Shoot, stolon and tuber formation on potato (Solanum tuberosum L.) cuttings in response to photoperiod. Plant Physiology, 61(3): 348-353.

11. FAO. 2009. Nueva luz sobre un tesoro enterrado: Año internacional de la papa 2008, Reseña De Fin De Ano. Food \& Agriculture Org.

12. FRAC. 2013. FRAC Code List (C * 2013 : Fungicides sorted by mode of action (including FRAC Code numbering). http://www.frac.info/ publication/anhang/FRAC Code List 2013-update April-2013, consulta en enero de 2014.

13. Gregory LE. 1956. Some factors for tuberization in the potato plant. American Journal of Botany, 43(4): 281-288.

14. Henfling JW. 1987. El Tizón tardío de la papa: Phytophthora infestans. Boletín de información técnica (Segunda ed.). Lima, Peru: International Potato Center.

15. Herrera CA, Fierro LH y Moreno JD. 2000. Manejo integrado del cultivo de la papa. Manual Técnico. Produmedio. p. 196. Mosquera, Cundinamarca: CORPOICA Regional Uno. C.I.Tibaitatá.
16. Jackson SD. 1999. Multiple signaling pathways control tuber induction in potato. Plant Physiology, 119(1): 1-8.

17. Jaramillo S. 2003. Monografía sobre Phytophthora infestans (Mont) de Bary. p. 141. Medellín, Colombia: Universidad Nacional de Colombia.

18. Karadimos DA, Karaoglanidis GS y TzaveIla-Klonari K. 2005. Biological activity and physical modes of action of the Qo inhibitor fungicides trifloxystrobin and pyraclostrobin against Cercospora beticola. Crop Protection, 24(1): 23-29.

19. Köhle H, Grossman K, Jabs T, Gerhard M, Kaiser W, Glaab J y Herms S. 2002b. Physiological effects of the strobilurin fungicide. En: L H, R PE, D H-W y S HD (Eds.), Modern Fungicides and Antifungal Compounds III, $16 \mathrm{p}$.

20. Köhle H, Grossmann K, Jabs T, Gerhard M, Kaiser W, Glaab J y Herms S. 2002a. Physiological effects of the strobilurin F500 on plants. Modern Fungicides and Antifungal Compounds III. AgroConcept, Bonn, Germany.

21. Mares DJ, Sowokinos JR y Hawker JS. 1985. Carbohydrate metabolism in developing potato tubers. p. 279-327. En: Li PH. (Ed.), Potato Physiology. Academic Press. Orlando, Florida.

22. Moreno U. 1985. Environmental effects on growth and development of potato plants. p. 481-501. En: Li PH. (Ed.), Potato Physiology. Academic Press. Orlando, Florida.

23. Navia Ó, Gandarillas A, Ortuño N, Meneses E y Franco J. 2012. Tizón de la papa 
(Phytophthora infestans) y Agricultura Sostenible: Integración de resistencia sistémica inducida y estrategias de manejo integrado. http://www.proinpa.org/phocadownload/ articulos/Papa/Oscar Navia_tizon papa.pdf, consulta octubre de 2014.

24. Parrado JA, Pereiro GA, Rodríguez AJ, Córdova EP, García EV, González SP y Hernández RL. 2010. Efectividad del fungicida pyraclostrobin+boscalid $(6,8 \%+13,6 \%)$ sobre Alternaria solani sorauer en el cultivo de la papa (Solanum tuberosum L.). Fitosanidad, 14(4): 253-255.

25. Rivera HF, Martínez EP, Osorio JA y Martínez E. 2010. Respuesta de biosurfactantes producidos por Pseudomonas fluorescens para el control de la gota de la papa Phytophthora infestans (Mont.) de Bary, bajo condiciones controladas. Corpoica, Ciencia y Técnología Agropecuaria, 11(1): 21-30.

26. Robledo-Esqueda M, Lozoya-Saldaña $H$ y Colinas-León T. 2012. Inducción de Defensa en Papa (Solanum tuberosum L.) contra Phytophthora infestans Mont. de Bary por Fungicidas. Interciencia, 37(9): 689-695.

27. Salisbury FB y Ross CW. 1992. Plant Physiology. Cuarta edición, p. 682. Belmont, California: Wadsworth Publishing Company.
28. Schnabel G y Crisosto CH. 2008. Seasonal applications of a pyraclostrobin and boscalid mixture do not impact same-year peach fruit quality attributes. Hort Technology, 18(4): 678-684.

29. Stutte GW, Yorio NC y Wheeler RM. 1996. Interacting effects of photoperiod and photosynthetic photon flux on net carbon assimilation and starch accumulation in potato leaves. Journal of the American Society for Horticultural Science, 121(2): 264-268.

30. Taiz L y Zeiger E. 2006. Plant Physiology. Cuarta Edición, p. 764. Sunderland, MA: Sinauer Associates.

31. Töfoli JG, Domingues RJ, Garcia $\bigcirc$ y Rodrigues MAT. 2003. Ação do fungicida pyraclostrobin+metiram no controle da requeima na cultura da batata. Horticultura Brasileira, 21(2):1-4 .

32. Valbuena R, Roveda G, Bolaños A, Zapata J, Medina C, Almanza P y Porras D. 2010. Escalas fenológicas de las variedades de papa Parda Pastusa, Diacol Capiro y Criolla "Yema de Huevo" en las zonas productoras de Cundinamarca, Boyaca, Nariño y Antioquia. CORPOICA. Mosquera, Cundinamarca: Produmedios.

33. Vega P. 2004. Evaluación de compuestos inductores de resistencia contra gota 
(Phytophthora infestans) en papa (Solanum tuberosum) en condiciones controladas. Universidad Pedagógica y Tecnológica De Colombia.

34. Viola R, Roberts AG, Haupt S, Gazzani S, Hancock RD, Marmiroli N y Oparka KJ. 2001. Tuberization in potato involves a switch from apoplastic to symplastic phloem unloading. The Plant Cell Online, 13(2): 385-398.

35. Wang Wen-qiao, Zhang P, Meng Run-jie, Zhao Jian-jiang, Huang Qi-liang, Han Xiuying, Ma Zhi-qiang y Zhang Xiao-feng. 2014. Fungitoxicity and synergism of mixtures of fluopicolide and pyraclostrobin against Phytophthora infestans. Crop Protection, 57: 48-56.

36. Wingsle G, Karpinski S y Hallgren J. 1999. Low temperature, high light stress and antioxidant defence mechanisms in higher plants. Phyton, 39(4): 253-268.

37. Xiangwei, Congyun, Fengmao, Yanping y Jiannan. 2012. Dissipation of pyraclostrobin and its metabolite BF-500-3 in maize under field conditions. Ecotoxicology and Environmental Safety, 80: 252-257. 